\title{
Estudio preliminar de las propiedades psicométricas de la escala para la identificación de la violencia y el ámbito donde se presenta para niños de 8 a 12 años (EVIN)
}

\author{
Preliminary study of the psychometric properties of the scale for the identification of \\ violence and the scope where it is presented for children from 8 to 12 years old
}

Nisi Parraguez Burga1*, Janeth Bendezú Olivares², Jania Jaimes Soncco ${ }^{3}$

\section{RESUMEN}

Objetivos: El objetivo de este estudio fue construir un instrumento en base a las propiedades psicométricas, con el fin de identificar la violencia de tipo físico, psicológico y sexual en los ámbitos de casa, calle y colegio. Materiales y Métodos: El diseño de la investigación es de enfoque cuantitativo, de diseño no experimental, corte transversal y de tipo psicométrico debido a que se buscó establecer la validez y confiabilidad del cuestionario diseñado para este estudio. Resultados: En la primera etapa se realizó la delimitación conceptual del constructo y se elaboró el instrumento preliminar (63 ítems) basado en la revisión de la bibliografía referente a la violencia en la niñez. Se valoró la calidad de los ítems a través de 6 expertos en el área utilizando el coeficiente de validez $\mathrm{V}$ de Aiken. En la segunda etapa se consideró una muestra piloto de 401 escolares en dos instituciones educativas, donde se realizó la selección de ítems en base a tres criterios estadísticos. Luego se procedió con el análisis factorial exploratorio a fin de someter a un análisis riguroso de la naturaleza teórica y dimensional del constructo. Además, se obtuvo el coeficiente alpha de Cronbach para las sub dimensiones y escala global. Conclusiones: La escala final fue constituida por 38 ítems que forman 3 dimensiones y 3 sub dimensiones que cuentan con adecuados niveles de fiabilidad y validez.

Palabras claves: Violencia, niñez, psicometría, físico, psicológico, sexual. (Fuente: DeCS BIREME)

\section{ABSTRACT}

Objective: The objective of this study was to construct an instrument based on psychometric properties, in order to identify physical, psychological and sexual violence in the home, street and school settings. Material and Methods: The design of the research is quantitative, non-experimental design, cross-section and psychometric because it sought to establish the validity and reliability of the questionnaire designed for this study. Results: In the first stage, the conceptual delimitation of the construct was carried out and the preliminary instrument (63 items) was elaborated based on a review of the literature on violence in childhood. The quality of the items was evaluated through 6 experts in the area using the coefficient of validity $V$ of Aiken. In the second stage, a pilot sample of 401 students was considered in two educational institutions, where the selection of items was performed based on three statistical criteria. Then, the exploratory factor analysis was carried out in order to rigorously analyze the theoretical and dimensional nature of the construct. In addition, the Cronbach alpha coefficient was obtained for the sub-dimensions, and global scale. Conclusions: The final scale was composed by 38 items that form 3 dimensions and 3 sub dimensions that have adequate levels of reliability and validity.

Keywords: Violence, childhood, psychometry, physical, psychological, sexual. (Source: MeSH NLM)

${ }^{1}$ Licenciado en Psicología. Consultoría privada, Lima, Perú.
${ }^{2}$ Docente de Psicología, Facultad de Ciencias de la Salud, Universidad Peruana Unión, Lima, Perú. 


\section{INTRODUCCIÓN}

La violencia es un problema de salud pública que está afectando profundamente a la sociedad y cada día se vienen incrementando los casos siendo evidenciado a través de las denuncias que realizan los agraviados, a través de las noticias en los periódicos, televisión, radio, internet, etc. (Fernández, 2014).

La Organización Mundial de la Salud (OMS, 2006) reveló que los menores de edad son los que corren más riesgo de sufrir violencia por algún familiar y/o de personas cercanas a ellos. En este sentido, definimos a la violencia infantil como el uso de la fuerza con intención y de manera reiterativa, hacia el niño y niña, dentro o fuera del círculo familiar realizado por el o la progenitor(a), persona encargada de su cuidado o cualquier adulto que haciendo uso de su autoridad y poder, amenace el desarrollo físico, psicológico y sexual del menor, causándoles graves daños y/o hasta la muerte (Aguilar, 1992; Asili y Pinzón, 2003; Gracia y Musitu, 1993; Loredo, 1994 y Castro y Criscuolo, 2014).

Además, el Instituto Nacional de Estadística e Informática (INEI, 2015), a través de la Encuesta Demográfica y de Salud Familiar (ENDES), identificó que el $19.8 \%$ de las entrevistas cree necesario castigar a sus hijos para educarlos, creen que causando cierto grado de dolor pueden imponer disciplina, control o modificar la conducta. Las principales formas que utilizan para corregir a sus hijos son la reprimenda verbal $(61,8 \%)$, el hablar con los menores y explicarles la conducta (35\%), el prohibirles algo que les gusta $(22,3 \%)$, el darles palmadas $(10,9 \%)$, golpes y castigos físicos $(14,7 \%)$.

En el Perú, son escasos los instrumentos válidos y confiables que permitan identificar la violencia, específicamente en la niñez en los diferentes lugares. Algunos de estos son los siguientes: la Ficha de Valoración de Riesgos utilizada por las dependencias policiales, el Centro Emergencia Mujer (CEM), entre otras entidades públicas. También está el Cuestionario de Tamizaje de Violencia y la Ficha Epidemiológica de Violencia Familiar, utilizados por el Ministerio de Salud. Estos instrumentos son aplicados tanto a niños como a los adultos, pero no tienen preguntas específicas ni entendibles para la infancia (Mesa de trabajo intersectorial contra el feminicidio, 2012).
Por último, esta investigación tuvo como objetivo desarrollar una escala para la evaluación de la violencia en la infancia "EViN", es concisa, de rápida aplicación, y que permite identificar el tipo de violencia física, psicológica o sexual; asimismo, el ámbito donde se ejecuta como la casa, el colegio y la calle.

\section{MATERIALES Y MÉTODOS}

El diseño de la investigación es de enfoque cuantitativo, de diseño no experimental, corte transversal y de tipo psicométrico debido a que se buscó establecer la validez y confiabilidad del cuestionario diseñado para este estudio (Barbero, 2010).

\section{Participantes}

Para la muestra piloto participaron niños de entre 8 a 12 años de edad de ambos sexos, del 3er al 6to grado del nivel primario de las Instituciones: I.E. No 1260 "El Amauta" y I.E. N 1245 “José C. Mariátegui”. Se hizo una selección de muestra por conveniencia. Después de una depuración de encuestas basada en los criterios de exclusión, se quedó con 401 encuestas.

\section{Instrumentos}

Se inició la escala con 63 ítems, los cuales identifican los tipos de violencia (físico, psicológico y sexual) y el ámbito donde se presenta (casa, colegio y calle). La escala pasó por el primer análisis mediante criterio de cinco jueces especialistas en el área. Con base en sus indicaciones y recomendaciones se modificaron algunos términos de los ítems para que sea más entendible por el menor. Posterior a las modificaciones, la escala estuvo lista para su aplicación a la muestra piloto. En seguida, se realizó el análisis estadístico donde se eliminó 25 ítems según criterios de correlación, alfa y análisis factorial, quedando 38 ítems. El tipo de respuesta es de tipo Likert de tres puntos, donde nunca $=1$ (nunca te pasó esto), algunas veces $=2$ (algunas veces te pasa esto) y todos los días $=3$ (todos los días te pasa esto). Se construyó esta escala en base al modelo ecológico de la violencia de Belsky (1993), Aguilar (1992), Asili y Pinzón (2003), Gracía y Musitu (1993), Loredo (1994), Castro y Criscuolo (2014), Kempe, Silverman, Steele, Droegemueller y Silver (1962), tomando en cuenta el constructo "Cuestionario de Exposición a la Violencia" de Orue y Calvete (2010). Esta escala ayuda en la 
identificación del tipo de violencia y el ámbito en donde se desarrolla, la cual se clasifica en 3 dimensiones y 3 ámbitos:

\section{a. Dimensiones, tipos de violencia}

- Violencia física: incluye golpes, palmadas, patadas, marcas y cicatrices, etc, cuyos ítems son: 1, 3, 10, 11, 12, 19, 20, 21, 29, 30, 37, 38, 39, 55, 56, 57.

- Violencia psicológica: incluye humillación, amenazas, burlas, indiferencia, rechazo y gritos, cuyos ítems son: 7, 8, 17, 18, 25, 26, $27,36,43,45,52,54,61,62,63$.

- Violencia sexual: incluye tocamientos indebidos, obligaciones sexuales, exhibicionismo y pornografía, dichos ítems son: 6,32 , $41,42,50,51,59$.

\section{b. Ámbitos de violencia}

- Ámbito casa: Ejercida por padres, hermanos, tíos, primos, abuelos, padrastros, madrastras, empleadas, cuidadoras. Los ítems que evalúa son: $3,6,12,18,21,27,30,36$, $39,42,45,51,54,57,63$.
- Ámbito colegio: Ejercida por compañeros del salón, compañeros de la escuela, profesores y autoridades. Los ítems que evalúa son: $1,7,10,19,25,37,43,52,55,61$.

- Ámbito calle: Ejercida por personas desconocidas, vecinos, pandilleros, callejeros. Los ítems que evalúa son: 8, 11, 17, 20, 26, 29, $32,38,41,50,56,59,62$.

\section{RESULTADOS}

\section{Fiabilidad preliminar de la escala}

Abad, Garrido, Olea y Ponsoda (2006), refieren que la fiabilidad es el grado de estabilidad, precisión o consistencia que manifiesta el test como instrumento de medición de un rasgo determinado. Para la fiabilidad preliminar de la escala de violencia (EViN), de sus dimensiones y sub dimensiones, se valoró calculando el índice de la consistencia interna mediante el coeficiente alpha de Crombach. Se puede observar en la Tabla 1 la consistencia interna global de violencia (63 ítems) la muestra estudiada es de 634, que puede ser valorado como indicador de buena fiabilidad.

Tabla 1

Primer análisis de consistencia interna de la Escala de violencia EViN.

\begin{tabular}{ccc} 
Dimensión & No ítems & Alpha \\
\hline Físico & 21 & .695 \\
Colegio & 7 & .565 \\
Casa & 7 & .518 \\
Calle & 7 & .854 \\
Psicológico & 21 & .555 \\
Colegio & 7 & .263 \\
Casa & 7 & .400 \\
Calle & 7 & .453 \\
Sexual & 21 & .573 \\
Colegio & 7 & .613 \\
Casa & 7 & .264 \\
Calle & 7 & .454 \\
Escala de violencia EVIN & 63 & .634 \\
\hline
\end{tabular}

Análisis factorial global de segundo orden

Para el análisis factorial global de segundo orden, se utilizó el método de componentes principales para auto valores fijos a 3. Así mismo, se utilizó el test de screening de Cattel para complementar el 
análisis anterior. Antes de la realización del análisis factorial global, se corroboró la matriz de datos, que no sea una matriz de identidad $(p<.5)$ y que el grado de correlación entre los ítems sea entre moderado y alto (.60) es ahí donde puede considerarse al análisis factorial exploratorio como una técnica apropiada. En la Tabla 2 se observa que todos las subdimensiones se agrupan según su peso factorial formando tres dimensiones: violencia en casa, violencia en colegio y violencia en la calle, cada dimensión con las sub dimensiones que le corresponde: violencia física, psicológica y sexual.

Tabla 2

Matriz de factores rotados para las 3 dimensiones y subdimensiones de la escala de violencia (EViN)

\begin{tabular}{llll} 
& \multicolumn{2}{l}{ Componente-Subdimensiones } & \\
\cline { 2 - 4 } Dimensiones & Calle & Casa & Colegio \\
Físico - Calle & .767 & .197 & .041 \\
Sexual - Calle & .730 & -.295 & .139 \\
Psicológico - Calle & .647 & .359 & .011 \\
\cline { 2 - 4 } Sexual - Casa & .433 & .073 & .082 \\
Psicológico - Casa & .055 & .839 & .098 \\
Físico - Casa & .173 & .839 & .836 \\
Psicológico - Colegio & -.015 & .178 & .836 \\
Físico - Colegio & .162 & .031 & \\
\hline \% Total de varianza explicada & & $61.75 \%$ & \\
Prueba de KMO & & .607 & \\
Prueba de Barlett & & $x^{2}(21)=530.914$ \\
\hline
\end{tabular}

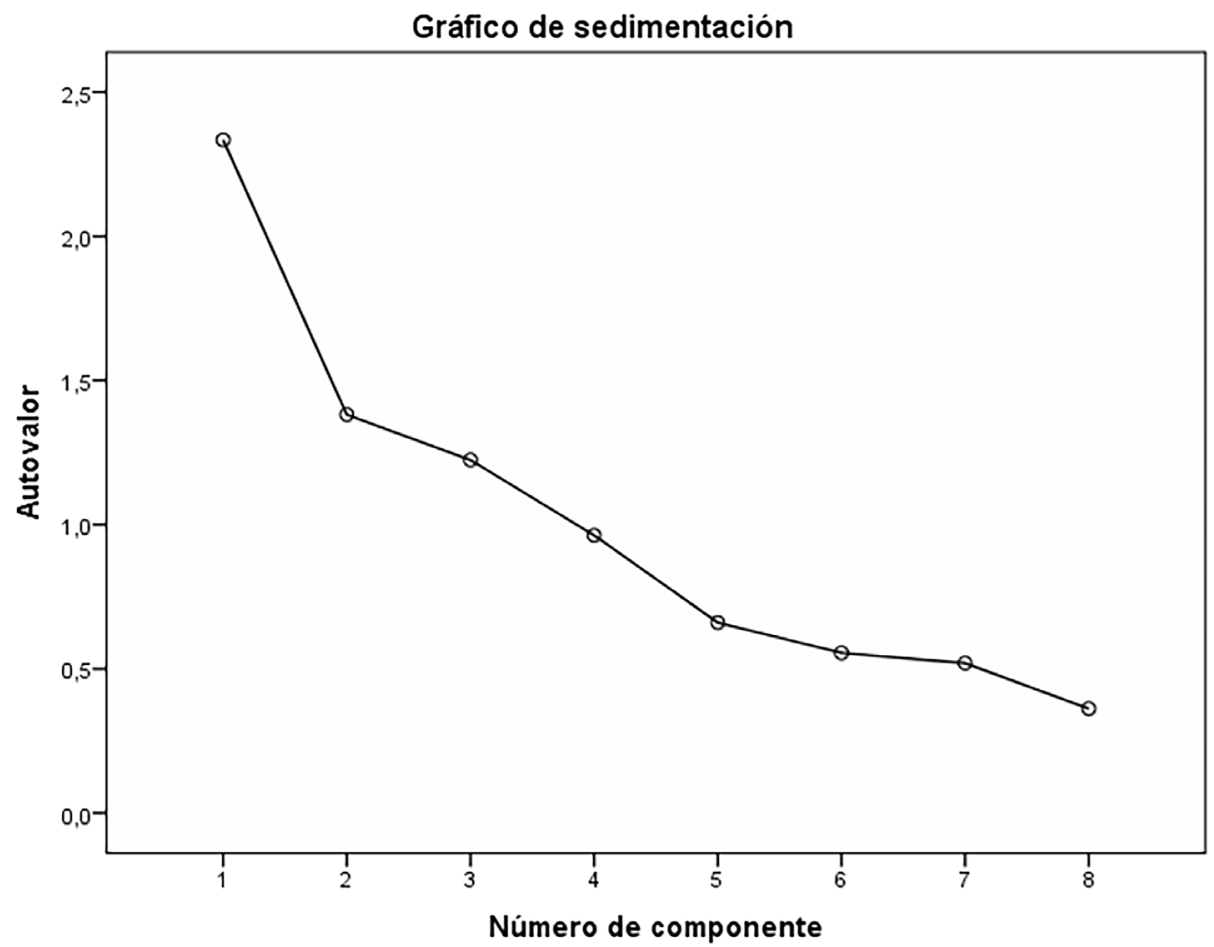

Figura 1. Gráfico de sedimentación de Cattell 


\section{Fiabilidad de la escala de violencia EViN}

Para la fiabilidad global de la escala de violencia (EViN), de sus dimensiones y sub dimensiones, se valoró calculando el índice de la consistencia interna mediante el coeficiente alpha de Crombach. Se puede observar en la Tabla 3, la consistencia interna global de violencia (38 ítems) en la muestra estudiada es de $\alpha=, 794$ que puede ser valorado como indicador de una buena fiabilidad. En relación a las dimensiones, se aprecia que, la dimensión física obtuvo un $\alpha=.674$, dimensión psicológica $\alpha=.677$ y dimensión sexual $\alpha=.619$. Estos resultados son considerados como indicadores de una buena fiabilidad para los instrumentos de medición psicológica (Gonzalvo, 1978; Kaplan \& Saccuzzo, 2006).

\section{Tabla 3}

Versión final de las estimaciones de consistencia interna de la Escala de Violencia EViN.

\begin{tabular}{lll} 
Dimensión & $N^{\circ}$ ítems & Alpha \\
\hline Físico & 16 & .674 \\
Colegio & 5 & .599 \\
Casa & 6 & .689 \\
Calle & 5 & .484 \\
Psicológico & 15 & .677 \\
Colegio & 5 & .612 \\
Casa & 6 & .625 \\
Calle & 4 & .522 \\
Sexual & 7 & .619 \\
Casa & 3 & .371 \\
Calle & 4 & .734 \\
Escala de violencia EVIN & 38 & .794 \\
\hline
\end{tabular}

Para la interpretación de las puntuaciones, se estableció puntajes directos en función a percentiles lo cual: a mayor puntaje obtenido mayor presencia de violencia, tipo de violencia y ámbito en el que se presenta. En la Tabla 4 y 5 se presenta los percentiles para la interpretación de las puntuaciones.

\section{Tabla 4}

Categorías de Dimensiones de la escala de violencia EViN

\begin{tabular}{llllll} 
& & Sexual & Psicológico & Físico & Global \\
Percentiles & 5 & 7,00 & 15,00 & 16,00 & 39,00 \\
& 10 & 7,00 & 15,00 & 16,00 & 39,00 \\
& 15 & 7,00 & 16,00 & 16,00 & 40,00 \\
20 & 7,00 & 16,00 & 17,00 & 41,00 \\
& 25 & 7,00 & 16,00 & 17,00 & 41,00 \\
& 30 & 7,00 & 17,00 & 17,00 & 42,00 \\
35 & 7,00 & 17,00 & 18,00 & 42,00 \\
\hline 40 & 7,00 & 17,00 & 18,00 & 43,00 \\
& 45 & 7,00 & 18,00 & 18,00 & 43,00 \\
50 & 7,00 & 18,00 & 18,00 & 43,00 \\
55 & 7,00 & 18,00 & 19,00 & 44,00 \\
60 & 7,00 & 19,00 & 19,00 & 45,00 \\
& 65 & 7,00 & 19,00 & 19,00 & 45,00 \\
70 & 7,00 & 19,00 & 20,00 & 46,00 \\
& 75 & 7,00 & 20,00 & 20,00 & 47,00 \\
80 & 7,00 & 20,00 & 20,60 & 47,00 \\
& 85 & 7,00 & 21,00 & 21,00 & 48,70 \\
90 & 7,00 & 22,00 & 22,00 & 50,00 \\
\hline 9 & 8,00 & 24,00 & 24,00 & 54,00 \\
\hline
\end{tabular}


Estudio preliminar de las propiedades psicométricas de la escala para la identificación de la violencia y el ámbito donde se presenta para niños de 8 a 12 años (EVIN)

Tabla 5

Categorías de Sub dimensiones de la escala de violencia EViN.

\begin{tabular}{|c|c|c|c|c|}
\hline & & Colegio & Casa & Calle \\
\hline \multirow[t]{19}{*}{ Percentiles } & 5 & 10,00 & 15,00 & 13,00 \\
\hline & 10 & 10,00 & 15,00 & 13,00 \\
\hline & 15 & 10,00 & 15,00 & 13,00 \\
\hline & 20 & 10,40 & 15,00 & 13,00 \\
\hline & 25 & 11,00 & 15,00 & 13,00 \\
\hline & 30 & 11,00 & 16,00 & 13,00 \\
\hline & 35 & 11,00 & 16,00 & 13,00 \\
\hline & 40 & 11,00 & 16,00 & 13,00 \\
\hline & 45 & 12,00 & 17,00 & 14,00 \\
\hline & 50 & 12,00 & 17,00 & 14,00 \\
\hline & 55 & 12,00 & 17,00 & 14,00 \\
\hline & 60 & 13,00 & 17,20 & 14,00 \\
\hline & 65 & 13,00 & 18,00 & 14,00 \\
\hline & 70 & 13,00 & 18,00 & 15,00 \\
\hline & 75 & 14,00 & 19,00 & 15,00 \\
\hline & 80 & 14,00 & 19,00 & 15,00 \\
\hline & 85 & 15,00 & 20,00 & 16,00 \\
\hline & 90 & 16,00 & 21,00 & 17,00 \\
\hline & 95 & 17,00 & 23,00 & 18,00 \\
\hline
\end{tabular}

En la Tabla 6 y 7, al tomar en cuenta los valores percentiles (Pc 25 y Pc 75) para los puntos de corte por dimensiones y sub dimensiones, la valoración de la identificación del tipo de violencia y el ámbito en donde se presentase establecen del siguiente modo.

Tabla 6

Categorías de tipo de violencia en la escala de violencia EViN.

\begin{tabular}{llll}
\multirow{2}{*}{ Puntuación directa } & \multicolumn{3}{c}{ Nivel de uso } \\
\cline { 2 - 4 } & Bajo & Medio & Alto \\
\hline Físico & $0-17$ & $18-20$ & $21-48$ \\
Psicológico & $0-16$ & $17-20$ & $21-45$ \\
Sexual & $0-6$ & $7-8$ & $9-21$ \\
TOTAL & $0-41$ & $42-47$ & $48-111$ \\
\hline
\end{tabular}

Tabla 7

Categorías del ámbito que se ejerce la violencia en la escala de violencia EViN

\begin{tabular}{llll}
\multirow{2}{*}{ Puntuación directa } & Nivel de uso & \\
\cline { 2 - 4 } & Bajo & Medio & Alto \\
\hline Colegio & $0-11$ & $12-14$ & $15-30$ \\
Casa & $0-15$ & $16-19$ & $20-45$ \\
Calle & $0-13$ & $14-15$ & $16-$ más \\
\hline
\end{tabular}




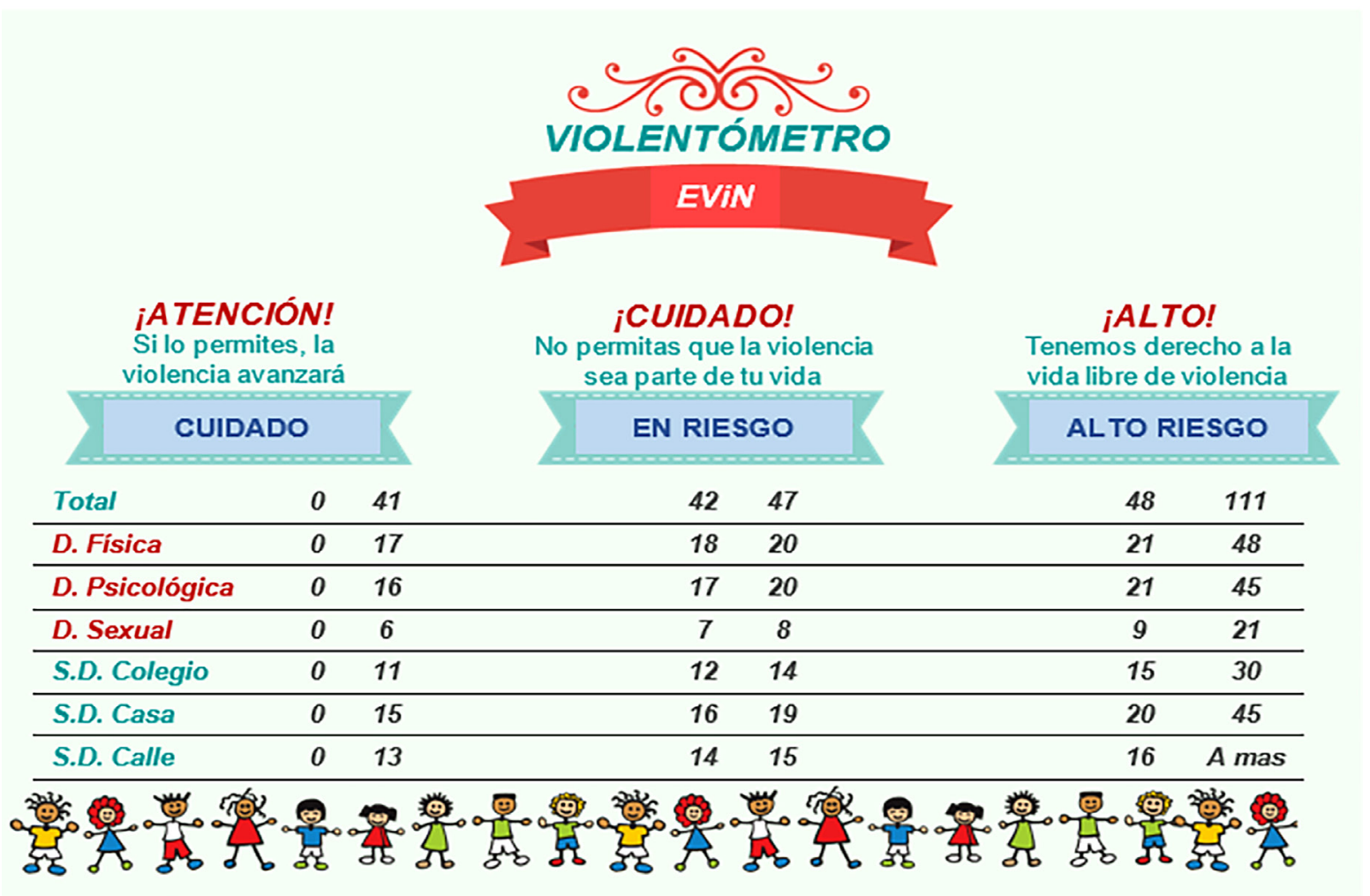

\section{DISCUSIÓN}

Los análisis psicométricos de este estudio brindan evidencias empíricas que respaldan el objetivo de construcción y validación de la escala de la violencia (EViN) en escolares. Esta escala es capaz de identificar la violencia en la niñez en toda su complejidad.

En relación a ello, después de un análisis teórico se elaboraron 63 ítems en relación al constructo, dimensiones y sub-dimensiones. Luego, se procedió a la validación de contenido, donde participaron 6 jueces expertos en el área (1 doctor, 2 magister y 3 licenciados), se utilizó, el coeficiente de validez $\mathrm{V}$ de Aiken para valorar la congruencia, claridad en la redacción, uso de palabras en el contexto y dominio del constructo. En la escala se modificó 7 ítems en cuanto a la claridad. Finalmente, se obtuvo 63 ítems claros, congruentes, en el contexto adecuado y con buen dominio de constructo.

Posterior a ello se realizó la coordinación con dos instituciones educativas estatales de Huaycán. La I.E. N 1260 "El Amauta” y la I.E. N 1245 "José C. Mariátegui". Se coordinaron dos fechas para la aplicación de la escala (EViN) a la muestra piloto, que es una pequeña proporción de la población con carácter homogéneo en la que se puede hacer inferencias en base a un estudio sobre la población (Gonzalvo, 1978). El análisis piloto contó 500 alumnos de 3ro, 4to, 5to y 6to grado de Primaria de instituciones educativas estatales de Huaycán.

En base a las pruebas aplicadas, se procedió a realizar la depuración de encuestas, donde se descartaron 99, quedando 401 pruebas, ya que tenían más del 10\% de preguntas omitidas, respuestas al azar, respuestas nulas y el $80 \%$ de respuestas homogéneas, para después elaborar la plantilla en el SPSS y hacer los siguientes análisis estadísticos.

El primer análisis fue la depuración y selección de ítems, cuyo criterio de selección fueron: a) correlación ítem sub-test, siendo la correlación de los ítems y la dimensión mayores de 0.5 (Barranca, López \& Olea, 2000). Según este criterio, se eliminaron 11 ítems de toda la escala; 2 ítems de la dimensión violencia física, 3 ítems de la dimensión violencia psicológica y 6 ítems de la dimensión violencia sexual puesto que no cumplieron con el criterio ya mencionado. b) El segundo criterio fue el coeficiente de alpha de crombach el cual sirve para medir la fiabilidad de una escala, el cual toma valores entre 0 y 
1 que sirven para comprobar si el instrumento está evaluando la variable que se quiere medir, para así tener un instrumento fiable con ediciones estables y consistentes (Gonzalvo, 1978). Con base en ese criterio, se eliminó 2 ítems de la dimensión violencia física y 8 ítems de la dimensión violencia sexual. Por último, c) el tercer criterio estadístico fue el análisis factorial por dimensión, por el cual los ítems se agruparían según su peso factorial y correlación. En el caso de los ítems con puntaje menor a .30, automáticamente fueron eliminados; en la dimensión física, se eliminaron 2 ítems, en la dimensión psicológica 3 ítems y en la dimensión sexual 6 ítems y 3 no fueron considerados ya que no cumplieron con los requisitos de este criterio.

El siguiente análisis estadístico fue el análisis factorial exploratorio de primer orden ítems y dimensiones, donde se utilizó el método de componentes rotados varimax para así poder tener una estructura interna según sus componentes. En la dimensión física, en el último análisis se evidenció la formación de 4 componentes, donde los ítems se agruparon adecuadamente en las tres dimensiones correspondientes, a excepción del ítem 20 que forma un 4to componente; sin embargo, también posee un peso adecuado en su propia dimensión. En la dimensión psicológica, de igual manera, se evidenció la formación de 4 componentes, en los cuales los ítems se agruparon correspondientemente a su componente, con excepción del ítem 17; al igual que el anterior, presenta un peso adecuado en el componente que le corresponde. Finalmente, en la dimensión sexual, en el último análisis se mostró la formación de 2 componentes, cuyos ítems fueron adecuadamente agrupados en dos dimensiones.

Seguidamente, se realizó el análisis factorial global exploratorio de segundo orden para tres dimensiones del instrumento donde se corroboró que la correlación de los ítems es moderado y alto (.60). Se utilizó el test screening de Cattel para seguir con el análisis, donde se encontró que las tres dimensiones explican la varianza adecuada del constructo; puesto que el gráfico de sedimentación determina el número óptimo de factores, explicando la varianza de los componentes principales.

La fiabilidad del constructo tuvo una consistencia interna mediante el coeficiente de alpha de crombach que hace referencia a la medición de la fiabilidad de la escala (Kaplan \& Saccuzzo, 2006). La escala global de violencia EViN presentó una adecuada fiabilidad, puesto que su consistencia interna es de $\alpha=, 794$; así mismo, en sus dimensiones presentaron: dimensión física obtuvo un $\alpha=.674$, dimensión psicológica $\alpha=.677$ y dimensión sexual $\alpha=.619$; es decir, el instrumento es apto para la medición de la violencia infantil ya que contiene una adecuada fiabilidad para la aplicación en la población peruana. Es necesario recalcar que este estudio es el primer análisis piloto que en su posterioridad se realizará el estudio confirmatorio.

Luego del análisis psicométrico, la escala de violencia "EViN" cuenta con 38 ítems, que permiten identificar el tipo de violencia ya sea física (16í), psicología (15í) y sexual (7í) percibida en los ámbitos de colegio, casa y calle. Esta escala cuenta con tres niveles para categorizar a la violencia infantil y son: cuidado (bajo), en riesgo (medio) y alto riesgo (alto) para el constructo global y sus dimensiones respectivas. Esta escala EViN es válida y confiable para ser utilizada en nuestro contexto social, se cumple así los objetivos planteados inicialmente.

Declaración de financiamiento y de conflictos de interés:

El estudio fue financiado por los autores, quienes declaran no tener conflictos de interés.

\section{Correspondencia}

Nisi Parraguez Burga. Dirección: Facultad de Ciencias de la Salud, Universidad Peruana Unión, Carretera central Km 19, Ñaña, Lima, Perú.

Correo electrónico: nisi_1895@hotmail.com 


\section{REFERENCIAS BIBLIOGRÁFICAS}

Abad, F., Garrido, J., Olea, J. \& Ponsoda, V. (2006). Introducción a la psicometría. Madrid: Universidad Autónoma de Madrid.

Aguilar, A. (1992). El maltrato de los niños y sus repercusiones educativas. Memorias del Segundo Simposium Interdisciplinario e Internacional. México: Federación Iberoamericana contra el Maltrato Infantil.

Almenares, M., Louro, I. \& Ortiz, M. (1999). Comportamiento de la Violencia Intrafamiliar. Revista Cubana Med Gen Integr, 15(3), 285292. Recuperado de http://scielo.sld.cu/pdf/mgi/ v15n3/mgi11399.pdf

Armeta, M. \& Gaxiola, J. (2008). Consecuencias de la violencia familiar experimentada directa e indirectamente en niños: depresión, ansiedad, conducta antisocial y ejecución académica. Revista Mexicana de Psicológica, 25(2), 237248. Recuperado de http://www.redalyc.org/ pdf/2430/243016308004.pdf.

Arruabarrena, I. \& De Paúl, J. (1999). Maltrato a los niños en la familia: evaluación y tratamiento. Madrid, España: Ediciones Pirámide.

Asili, P. \& Pinzón L. (2003). Percepción de los estilos parentales y bienestar psicológico. Psicología y Salud, 2(12), 215-227.

Baider, L., Peretz, Handani, P., Perry, S., Avramv, R. \& Kaplan de Nour, A. (2000). Transmission of response to trauma? Second-generation holocaust survivors reactions to cancer. Am J Psychiatry, 157(9), 4-6. Recuperado de http:// www.ncbi.nlm.nih.gov/pubmed/10831469.

Bandura, A. \& Walters, R. (1973). Teoría del aprendizaje social y desarrollo de la personalidad. Madrid, España: Alianza Editorial.

Barlow, J., Johnston, I., Kendrick, D., Polnay, L. \& Stewart, B. (2008). Programas de parentalidad individuales y grupales para el tratamiento del abandono y el maltrato físico infantil. Revista Médica Electrónica, 35(6). Recuperado de http:// scielo.sld.cu/pdf/rme/v35n6/tema04.pdf.

Barbero, M. (2010). Psicometría. Madrid, España: Sanz y Torres.

Barranca, J., López, L. \& Olea, J. (2000). Psychometric properties of a new family life satisfaction scale. European journal of psychological assessment, 16(2), 98-106. Recuperado de http://www. academia.edu/4441397/Family_Satisfaction_ Scale.
Benenson, P. (2008). Escuelas seguras, el derecho de cada niña. España: Amnistia Internacional. Recuperado de https://docs. google.com/viewerng/viewer?url= http://www. observatorioperu.com/textos\%25202011/ Escuelas\%2520seguras.pdf\&hl=es.

Cantón, D. \& Cortes, M. (2015). Consecuencias del abuso sexual infantil: una revisión de las variables intervinientes. Anales de psicología, 41(2), 552561. Recuperado de http://www.redalyc.org/ pdf/167/16738685018.pdf.

Carreño, P. (2011). Comportamiento de la violencia intrafamiliar. Instituto Nacional de Medicina Legal y Ciencias Forenses. Bogotá, Colombia.

Castro, D. \& Criscuolo, E. (2014). Síndrome del niño maltratado. Intra Med Journal, 3(1). Recuperado de http://journal.intramed.net/index.php/ Intramed_Journal /article/viewFile/280/120.

Condori, M. \& Guerrero, R. (2010). Factores individuales, sociales y culturales que influyen en la violencia basada en género en mujeres de 20 a 64 años de edad en el centro de salud Ganimedes San Juan de Lurigancho. (Tesis para licenciatura, Universidad Nacional Mayor de San Marcos, Lima). Recuperado de http://cybertesis. unmsm.edu.pe/bitstream/cybertesis/2993/1/ Condori_fm.pdf.

Contreras, J., Bott, S., Guedes, A. \& Dartnall, E. (2010). Sexual violence in Latin America and the Caribbean: A deskreview. Marzo 2010. Sexual Violence Research Iniciative. Recuperado de https://www.ciaonet.org/attachments/19595/ uploads.

Echeburria, E. \& Guerricaechevarría, C. (2000) Abuso sexual en la infancia: víctimas y agresores. Madrid, España: Ariel.

El peruano. (30 de diciembre del 2015). Ley Nº 30403. p. 1.

Escalante, M. \& Uribe, R. (2001). Maltrato infantil. Temas de revisión 2001, 1(1) 48-50.

Fernández, G. \& Perea, J. (2004). Síndrome del maltrato infantil. Revista Cubana Pediátrica, 76(3). Recuperado de http:// scielo.sld.cu/scielo.php?pid=S0034$75312004000300009 \& \mathrm{script}=\mathrm{sci}$ arttext\&tIng=en.

Ferreira, A., Beltran, M., Montoya, C., Núñez, O. \& Bossio, J. (2013). Maltrato infantil y abuso sexual en la niñez. Brasil: AIEPI. 
Finkelhor, D., Hamby, S., Ormrod, R. \& Turner, H. (2005). The Juvenile Victimization Questionnaire: Reliability, validity and national norms. Child Abuse y Neglect, 29(4), 383-412.

García, G. (2013). Antecedente de violencia doméstica y actitud violenta en hombres residentes en Manchay, Lima. (Tesis para licenciatura, Universidad Ricardo Palma, Lima). Recuperado de http://cybertesis.urp.edu.pe/ bitstream/urp/307/1/ Garcia_gn.pdf.

García, C., Guedes, A. \& Knerr, W. (2013). Violencia Sexual. Comprender y abordar la violencia contra las mujeres, 1(1), 1-12. Recuperado de http:// www.who.int/reproductivehealth/publications/ violence/rhr12_37/es/.

Instituto nacional de estadística e informática. (2015). Encueta demográfica y de salud familiar ENDES. Recuperado de https://www.inei.gob.pe/ media /MenuRecursivo/publicaciones_digitales/ Est/Lib1211/pdf/Libro.pdf.

Loredo, A. (1994). Maltrato al menor. México: Mc Graw-Hill.

Méndez, C. y Rondón, M. (2012). Introducción al análisis factorial exploratorio. Revista colombiana de investigación, 41(1), 197-207. Recuperado de http://www.redalyc.org/articulo. oa?id=80624093014.

Mesa, P. \& Moya, L. (2011). Neurobiología del maltrato infantil: el ciclo de la violencia. Rev Neurol, 52, 489-503.

Ministerio de Educación (2012). Resolución Ministerial $\mathrm{N}^{\circ}$ 0519-2012-ED, 19 de diciembre, 2012, p.5. Recuperado de http://www.minedu.gob.pe/ normatividad/resoluciones.php.

Ministerio de la Mujer y Poblaciones Vulnerables. (2014). Estadísticas sobre violencia familiar y sexual, violencia contra la mujer y feminicidio en el Perú. Lima, Perú.

Ministerio de Salud (2014). Resolución Ministerial $N^{\circ}$ 917-2014-MINSA, 27 de noviembre del 2014, p. 69. Recuperado de ftp://ftp2.minsa.gob.pe/ normaslegales/2014/RM917-2014-MINSA.pdf.

Mojarro, M. (2006). Guía para el Diagnostico Presuntivo del maltrato Infanto-Juvenil. Centro Nacional de Equidad de Género y Salud Reproductiva, 1, 4-42.

Olivares, E. \& Inchaustegui, T. (2011). Modelo ecológico para una vida libre de violencia de género. México: Comisión Nacional para Prevenir y Erradicar la Violencia contra las Mujeres.
Recuperado de http://cedoc.inmujeres.gob.mx/ Igamvlv/MoDecoFinalPDF.pdf.

Organización Mundial de la Salud. (2011). Violencia contra la mujer: violencia de pareja y violencia sexual contra la mujer. Nota descriptiva $\mathrm{N}^{\circ} 239$. Actualización de septiembre de 2011. Ginebra, Organización mundial de la Salud.

Organización de las Naciones Unidas. (2014). Datos básicos: Estadísticas sobre la violencia contra las mujeres y niñas. Junio 2014, de ONU Sitio web: http://www.endvawnow.org/es/articles/299-datosbasicos-.html.

Orjuela, L \& Rodríguez, V. (2012). Violencia sexual contra los niños y las niñas. Abuso y explotación sexual infantil. España: Save the children Recuperado de https://www.savethechildren.es/ sites/default/files/imce/docs/violencia_sexual_ contra_losninosylasninas.pdf.

Orue, I. \& Calvete, E. (2012). Elaboración y validación de un cuestionario para medir la exposición a la violencia en infancia y adolescencia. International Journal of Psychology and Psychological Therapy, 10(2), 279-292. Recuperado de http:// www.ijpsy.com/volumen10/num2/262/elaboracin$y$-validacin-de-un-cuestionario-ES.pdf.

Papalia, D. \& Wendkos, S. (1997). Desarrollo humano. México: Mc Graw-Hill.

Policía Nacional del Perú. (2014). Estadísticas sobre violencia familiar y sexual, violencia contra la mujer y feminicidio en el Perú. Lima, Perú.

Ponce, J. (20 julio del 2016). Más de 8 mil menores han sido víctima de violencia en el año. El comercio. Recuperado de http://elcomercio.pe/ sociedad/peru/mas-8-mil-menores-han-sidovictimas-violencia-ano-noticia-1918145.

Straus, M., Hamby, S., Finkelhor, D., Moore, D. \& Runyan, D. (1998). The Resived Conflict Tactics Scales. Journal of Family Issaues, 17(3), 283316. Recuperado de https://pubpages.unh. edu/ mas2/CTS15.pdf.

Tarullo, A. (2012). Efects of child maltreatment on the developing brain. Child Welfare $360^{\circ}$ : Using a Developmental Approach in Child Welfare Practic, 11. Recuperado de https://www.bu.edu/ cdl/files/2013/08/Tarullo-CW360-2012.pdf.

Van, N. (2002). La formación del carácter y la personalidad, Hijos triunfadores. Colombia: Printed colombiana.

Vilca, L. \& Gonzales, A. (2013). Propiedades psicométricas de la Escala de Riesgo de 
Adicciones a las redes sociales. (Tesis para licenciatura). Universidad Peruana Unión, Lima.

White, E. (1964). Conducción del niño. Buenos Aires, Argentina: ACES.

White, E. (2007). El hogar cristiano. Buenos Aires, Argentina: ACES.

White, E. (1855). Joyas del testimonio 1. Buenos Aires, Argentina: ACES.

White, E. (1971). Consejo para los maestros. Buenos Aires, Argentina: ACES.

World Health Organization (2010). Addressing violence against women and HIVIAIDS. What works? Report of a consultation. Geneva: World Health Organization and Joint United Nations Programme on HIVIAIDS (UNAIDS). Recuperado de http://apps.who.int/iris/ bitstream/10665/44378/1/9789241599863_eng. pdf.
Zatzick, D., Kang, S., Moller H., Russo, J., Rivanc F., Katon, et al. (2002). Predicting posttraumatic distress in hospitalized trauma survivors with acute injuries. Am J Psychiatry, 159(94), 1-6. Recuperado de http://www.nctsnet.org /nctsn_ assets/Articles/121.pdf.

Zurbano, J. (2001). Educación para la convivencia y la paz. España: Gobierno de Navarra. Departamento de Educación y Cultura. Recuperado de https://docs.google.com/viewerng/ viewer?url=http://www.observatorioperu.com/ textos \%25202011/28022011/Educacion para_la_convivencia\%2520_y_para_la_ paz $\% 2520$ (primaria).pdf\&hl=es.

Recibido: $20 / 05 / 17$

Aceptado: 08/09/17 
La Revista Científica de Ciencias de la Salud, publica artículos originales empíricos y teóricos, comunicaciones originales cortas, reportes de casos en las áreas de Enfermería, Psicología, Nutrición y Medicina. Todo investigador que desee colaborar en la revista deberá considerar las siguientes indicaciones:

1. Los manuscritos deben enviarse a la revista en línea ingresando: http://revistascientificas. upeu.edu.pe/index.php/rc_salud, o escribir al correo electrónico: revistasalud@upeu. edu.pe. Deberán ser originales e inéditos y se asume que toda persona que figure como autor ha dado su conformidad para que el manuscrito sea publicado.

2. Los manuscritos recibidos para publicación son sometidos a un proceso de revisión por pares. El manuscrito es enviado a dos revisores nacionales o extranjeros, pares de los investigadores, quienes realizan las correcciones $u$ observaciones que sean pertinentes; luego el manuscrito es devuelto a los autores para que realicen las correcciones o respondan a las observaciones realizadas. En el caso que los revisores emitan opiniones contradictorias, se envía a un tercer revisor. Luego, el manuscrito es sometido a una revisión de la redacción y finalmente pasa a la diagramación. La versión diagramada tal como será publicada se envía a los autores para su corrección o aprobación.

3. El manuscrito debe ser presentado mediante una carta dirigida al Editor Jefe de la Revista Científica de Ciencias de la Salud, firmada por uno de los autores, solicitando la evaluación para ser considerada su publicación. La carta debe incluir el título del trabajo, el nombre completo de los autores y tipo de trabajo. Junto con la carta de presentación se debe entregar un original impreso, y la versión electrónica se debe enviar a la página web http://revistasinvestigacion.upeu.edu.pe/ index.php?journal=rc_salud

4. Se debe adjuntar una declaración jurada firmada por todos los autores (DECLARACION JURADA Autores Artículos), que el artículo presentado es propiedad intelectual de los autores, que no ha sido publicado, ni presentado para evaluación en otra revista, cediendo los derechos de autor a la Revista Científica de Ciencias de la Salud una vez que el artículo sea publicado.
5. El artículo debe ser redactado en castellano, portugués o inglés, impreso en papel bond blanco de medida ISOA4 (212x297 mm), en una sola cara, tipo de letra Times New Roman, tamaño de fuente 12 picas, a doble espacio, con márgenes de $25 \mathrm{~mm}$. Cada parte del artículo debe empezar en página aparte, numeradas en forma consecutiva. En el caso de citas textuales, el párrafo correspondiente dentro del texto se destacará colocándolo entre comillas o escribiéndolo con letra itálica. Las tablas y figuras con su título correspondiente, se colocan al final del texto en páginas aparte; no deben ser insertados en el texto.

\section{ESQUEMAS DE PRESENTACIÓN:}

Investigaciones originales: Resumen; Abstract; Introducción; Material y métodos; Resultados; Discusión; Referencias bibliográficas; Tablas y figuras. La extensión total del manuscrito, incluyendo las referencias bibliográficas, no debe ser mayor de 14 páginas escritas en una sola cara sin incluir tablas, gráficos y figuras. Se acepta como máximo de diez tablas y figuras; el número máximo de referencias bibliográficas es 40 .

\section{Comunicaciones cortas:}

Contienenlas mismas partes que unainvestigación original. La extensión total del manuscrito, incluyendo las referencias bibliográficas, no debe ser mayor de 6 páginas escritas en una sola cara, sin incluir tablas y figuras. Se acepta como máximo de seis tablas y figuras; el número máximo de referencias bibliográficas es 20.

\section{Reporte de casos:}

Resumen, Abstract; Introducción, Presentación del caso, Conclusiones, Referencias bibliográficas. La extensión total del trabajo, incluyendo las referencias bibliográficas, no debe ser mayor de seis páginas escritas en una sola cara, sin incluir tablas, gráficos y figuras. Se acepta como máximo de seis tablas, gráficos y figuras; el número máximo de referencias bibliográficas es 20 .

7. Los resúmenes (en el idioma original e inglés) se presentan cada una en hoja aparte, teniendo una extensión máxima de 250 palabras y deben ser escritos en un solo párrafo. Al final se deben agregar 3 a 5 palabras clave o key words, que ayuden a clasificar el artículo. 
8. La información a los autores e instrucciones para la presentación de manuscritos, donde se detallan las normas de formato y contenido de los artículos que se presentarán a la Revista, se pueden obtener de la página electrónica: http://revistasinvestigacion.upeu. edu.pe/index.php?journal=rc_salud

9. El estilo de la revista se basa en la $6^{\circ}$ edición revisada del Manual de Publicaciones de la APA.

10.Una vez que el autor ha sido notificado acerca de la aprobación de su trabajo para su publicación, no se administrarán modificaciones ni agregados.
11. Los nombres y direcciones de correo-e introducidos en esta revista se usarán exclusivamente para los fines declarados por esta revista y no estarán disponibles para ningún otro propósito u otra persona.

12.Política de acceso abierto: Esta revista provee acceso libre inmediato a su contenido bajo el principio de que hacer disponible gratuitamente la investigación al público, siempre y cuando cite correctamente dentro de su manuscrito, el articulo consultado, lo cual fomenta un mayor intercambio de conocimiento global. 